\title{
Cytokine Storm Syndrome: What Every Physician must Know Today?
}

\author{
Sagar Bhattad \\ Pediatric Infectious Disease (2020): 10.5005/jp-journals-10081-1251
}

\section{INTRODUCTION}

The term "cytokine storm" was first used in 1993 by Ferrara et al. to describe graft vs host disease in the setting of transplantation. ${ }^{1}$ Subsequently, cytokine storm has been implicated in the causation of severe viral infections, autoimmune and hematologic diseases, and adverse reaction to few drugs. With the current coronavirus disease-2019 (COVID-19) pandemic, there has been a renewed interest not only among the medical fraternity but in the lay public regarding this entity. Though several papers are being published on cytokine storm and its relevance to the current pandemic, it must be noted that there exists no clear definition as to what constitutes a "cytokine storm" and despite our growing understanding of this entity, targeting the storm with immunomodulation has not always yielded the desired outcomes. This article will provide an overview of "cytokine storm" and describe this entity in a simplified manner.

\section{What are Cytokines?}

Molecules that communicate among cells of the immune system are referred to as cytokines. Chemokines are low molecular weight proteins with powerful chemoattractant activity which play a role in the immune cell recruitment during inflammation.

\section{What is Cytokine Storm?}

"Excessive or uncontrolled release of inflammatory cytokines in the context of infectious and noninfectious diseases, and the accompanying immunopathology and the clinical consequences."

"An uncontrolled overproduction of soluble markers of inflammation which, in turn, sustain an aberrant systemic inflammatory response."

\section{Major Categories}

- Infection associated:

- Secondary hemophagocytic lymphohistiocytosis (HLH) associated with viral infections such as Epstein-Barr virus (EBV), cytomegalovirus (CMV), etc.

- Acute respiratory distress syndrome (ARDS) associated with COVID-19.

- Toxic shock syndrome: associated with staphylococcal or streptococcal infection.

- Autoimmune/autoinflammatory diseases: systemic onset juvenile idiopathic arthritis (SoJIA), Kawasaki disease, systemic lupus erythematosus. Note the macrophage activation syndrome (MAS) is the term used to describe the cytokine storm in rheumatic diseases.
Division of Pediatric Immunology and Rheumatology, Department of Pediatrics, Aster CMI Hospital, Bengaluru, Karnataka, India

Corresponding Author: Sagar Bhattad, Division of Pediatric Immunology and Rheumatology, Department of Pediatrics, Aster CMI Hospital, Bengaluru, Karnataka, India, Phone: +91 9779433934, e-mail: drsagarbhattad@gmail.com

How to cite this article: Bhattad S. Cytokine Storm Syndrome: What Every Physician must Know Today? Pediatr Inf Dis 2020;2(2): 79-81.

Source of support: Nil

Conflict of interest: None

- Malignancy: Associated HLH

- latrogenic: Car-T cell therapy in patients with leukemia and lymphoma.

\section{What are the Cytokines Implicated in this SYNDROME?}

IL-1, IL-6, TNF- $a$, INF- $\gamma$.

It must be noted that the predominant cytokine responsible for causing the systemic inflammation and multiorgan dysfunction may be different based on the underlying condition. This may in turn guide the targeted anticytokine therapy in a given patient. For example, IL- 6 is believed to be a key mediator for the cytokine storm in COVID-19 and, hence, the role of tocilizumab, an anti-IL-6R monoclonal antibody. Emapalumab, an anti-INF- $\gamma$ monoclonal antibody, has been approved for the treatment of refractory $\mathrm{HLH}$, because INF- $\gamma$ is the key cytokine driving the storm in the primary and secondary $\mathrm{HLH}^{2}$

\section{Pathophysiology}

Several models and hypotheses exist that explain the pathogenesis of cytokine storm:

\section{Super-antigen Theory}

T-cells get activated only when a specific antigen bound to MHC class II binds to the T-cell receptor (TCR), resulting in the activation of a specific clone of T-cells, that proliferate and handle the particular antigen. However, certain bacteria produce super-antigens (staphylococcal toxins) that bind to TCR V $\beta$ region. This binding is nonspecific and results in the activation of massive number of T-cells (up to $20 \%$ of total T cells in the body!) resulting in massive outpouring of cytokines, which in turn causes the cytokine storm

() The Author(s). 2020 Open Access This article is distributed under the terms of the Creative Commons Attribution 4.0 International License (https://creativecommons. org/licenses/by-nc/4.0/), which permits unrestricted use, distribution, and non-commercial reproduction in any medium, provided you give appropriate credit to the original author(s) and the source, provide a link to the Creative Commons license, and indicate if changes were made. The Creative Commons Public Domain Dedication waiver (http://creativecommons.org/publicdomain/zero/1.0/) applies to the data made available in this article, unless otherwise stated. 
syndrome (CSS). Toxic shock syndrome secondary to staphylococcal or streptococcal infections is a classical example.

\section{Defects in the Cytolytic Pathway}

CD8 T-cells (cytotoxic T-cells) and natural killer (NK) cells play an important role in clearing intracellular infections in our body, e.g., viral infections. This process involves killing of infected cells by releasing perforin and granzymes (the cytolytic pathway). A defect in this pathway results in a massive production of cytokines (predominant player being INF- $\gamma$ ), often triggered by viral infections, causing HLH.

\section{Defects in the Innate Immune Pathways}

Toll-like receptors (TLRs) are the pattern-recognition receptors of the innate immune system and play a pivotal role in identification and clearance of pathogens. Defects in TLR signaling pathways can result in aberrant activation of the immune system causing CSS. $^{3-5}$

\section{Immune Deficiency}

Patients with primary immune deficiencies fail to clear pathogens. Persistent infections trigger cytokine release and cause HLH. For example, patients with X-linked lymphoproliferative syndrome (XLP) develop HLH on exposure to EBV infection.

\section{Why Cytokine Storm in a Few?}

It is interesting to note, though several individuals develop infections with viruses, only a few of them develop the cytokine storm, implying host factors play an important role. Defects in the TLR signaling pathways and cytolytic pathways (host factors) are important determinants of the risk of cytokine storm in a given patient.

\section{Clinical Features}

High-grade fever is a consistent feature. This may be associated with features of systemic inflammatory response syndrome (SIRS): tachycardia, tachypnea, poor peripheral perfusion, and hypotension. In certain situations (e.g., COVID-19-associated cytokine storm), ARDS may be the prominent feature. In severe forms, multiorgan dysfunction including hepatic and renal failure may ensue, causing mortality, if not intervened in time. Differentiating a cytokine storm/ $\mathrm{HLH}$ and sepsis is challenging as many of the clinical features overlap. In this context, the role of $\mathrm{H}$-score is being discussed in the next section of this review.

\section{Laboratory Markers}

There is no single diagnostic test for the cytokine storm or $\mathrm{HLH}$. Bicytopenia or pancytopenia is often a finding (characteristic of HLH). Liver dysfunction (elevated liver enzymes and coagulopathy) may be seen. C-reactive protein (CRP) is markedly elevated and reflects IL-6-mediated inflammation. Ferritin $>10,000 \mathrm{ng} / \mathrm{mL}$ is often a diagnostic of $\mathrm{HLH}$, while the degree of hyperferritinemia may vary in other forms of cytokine storm syndromes.

\section{Sepsis or Cytokine Storm?}

As previously discussed, it is often challenging to differentiate sepsis and cytokine storm in a given clinical scenario. Use of " $\mathrm{H}$-score" may guide a clinician in such a scenario. A score $>120$ predicts $\mathrm{HLH}$ in children with sensitivity of $100 \%$ and specificity of $80 \%{ }^{6}$

\section{Relevance to Coronavirus Disease-2019}

A proportion of patients with COVID-19 develop severe respiratory illness warranting oxygen therapy and artificial ventilation in the most severe forms. Though several hypotheses have been proposed to explain the respiratory illness, ARDS seems to be the most plausible explanation. Patients with severe COVID-19 illness and ARDS have been shown to have markedly elevated IL- 6 levels compared to those with a milder disease, suggesting cytokine storm to be operative in this setting. ${ }^{7}$ In a recently published metaanalysis, a comparison of mean serum IL- 6 for severe COVID-19 and nonsevere COVID-19 was performed in seven studies. The mean serum IL-6 was $56.8(41.4-72.3 \mathrm{pg} / \mathrm{mL})$ and $17.3 \mathrm{pg} / \mathrm{mL}(13.5-21.1 \mathrm{pg} /$ $\mathrm{mL}$ ) for severe and nonsevere COVID-19 group, respectively. This was statistically significant $(p<0.001)$, and authors suggest a cut-off of more than $55 \mathrm{pg} / \mathrm{mL}$ for identifying patients at high risk of severe COVID-19. ${ }^{8}$

In line with these findings, several trials of tocilizumab, an IL-6 receptor-blocking monoclonal antibody, are underway. While uncontrolled studies involving a few patients seem to show some benefits in severe COVID-19 patients, ${ }^{9}$ results from controlled trials may offer definitive answers.

\section{Diagnosis}

One must suspect a cytokine storm/HLH in a patient in the presence of ongoing fever, features of SIRS, evolving or worsening cytopenia, rising CRP, and ferritin levels. The measurement of IL- 6 levels has been used to guide therapy in few studies. Clinical features and laboratory parameters along with a sensible application of $\mathrm{H}$-score would help in arriving at a timely diagnosis of CSS.

\section{THERAPY}

\section{Treat the Underlying Disease}

In patients with infection-associated $\mathrm{HLH}$, treating the underlying infection remains the cornerstone of the management. For example, EBV-associated HLH, injection Rituximab (anti-CD20 monoclonal antibody), is the treatment of choice to clear EBV infection along with immunomodulation for $\mathrm{HLH}$.

In patients with genetic forms of HLH (primary HLH), bone marrow transplantation would be curative.

\section{Immunomodulation}

Immune dysregulation is the cause of cytokine storm, and hence, immunomodulation plays a key role in the treatment of CSS.

- Steroids: intravenous pulse methylprednisolone $(10-30 \mathrm{mg} / \mathrm{kg} /$ day) for 3-5 days followed by oral steroids remains the treatment of choice in MAS noted in rheumatic diseases (e.g., SoJIA complicated with MAS). In case of severe/refractory disease, cyclosporine is added.

- Chemotherapy (dexamethasone + cyclosporine + etoposide) in severe forms of HLH (primary > secondary).

- Intravenous immunoglobulin (IVIg) is useful in cases of infectionassociated HLH.

- Cytokine-targeted therapy:

- Tocilizumab (anti-IL-6R antibody): If IL-6 is the key pathogenic cytokine. For example, cytokine release syndrome due to Car-T-cell therapy in leukemia and ARDS in COVID-19. 
- Emapalumab (anti-INF- $\gamma$ antibody): Refractory HLH.

- Anakinra (anti-IL-1R antibody): MAS in SoJIA.

\section{ConcLusion}

Cytokine storm syndrome is not a single disease but a group of disorders characterized by a massive production of cytokines that cause aberrant inflammation. A wide variety of clinical conditions may present with CSS, and better understanding of the pathophysiology has opened up newer avenues of cytokinetargeted therapies in these conditions. Further research in this direction will not only help us treat severe COVID-19 patients, which is the need of the hour, but would go a long way in treating many diseases where CSS seems to play a pivotal role.

\section{References}

1. Ferrara JL, Abhyankar S, Gilliland DG. Cytokine storm of graft-vshost disease: a critical effector role for interleukin-1. Transplant Proc 1993;25(1 Pt 2):1216-1217.

2. Locatelli $F$, Jordan $M B$, Allen $C$, et al. Emapalumab in children with primary hemophagocytic lymphohistiocytosis. N Engl J Med 2020;382(19):1811-1822. DOI: 10.1056/NEJMoa1911326.
3. Totura AL, Whitmore A, Agnihothram S, et al. Toll-like receptor 3 signaling via TRIF contributes to a protective innate immune response to severe acute respiratory syndrome coronavirus infection. mBio 2015;6(3):e00638-15. DOI: 10.1128/mBio.00638-15.

4. Gralinski LE, Menachery VD, Morgan AP, et al. Allelic variation in the toll-like receptor adaptor protein Ticam2 contributes to SARScoronavirus pathogenesis in mice. G3 (Bethesda) 2017;7(6):1653-1663. DOI: 10.1534/g3.117.041434.

5. Sheahan T, Morrison TE, Funkhouser W, et al. MyD88 is required for protection from lethal infection with a mouse-adapted SARS-CoV. PLoS Pathog 2008;4(12):e1000240. DOI: 10.1371/journal.ppat.1000240.

6. Debaugnies F, Mahadeb B, Ferster A, et al. Performances of the $\mathrm{H}$-score for diagnosis of hemophagocytic lymphohistiocytosis in adult and pediatric patients. Am J Clin Pathol 2016;145(6):862-870. DOI: 10.1093/ajcp/aqw076.

7. Coperchini F, Chiovato L, Croce L, et al. The cytokine storm in COVID19: an overview of the involvement of the chemokine/chemokinereceptor system. Cytokine Growth Factor Rev 2020;53:25-32. DOI: 10.1016/j.cytogfr.2020.05.003.

8. Aziz M, Fatima R, Assaly R. Elevated interleukin- 6 and severe COVID19: a meta-analysis. J Med Virol 2020. DOI: 10.1002/jmv.25948.

9. Luo P, Liu Y, Qiu L, et al. Tocilizumab treatment in COVID-19: a single center experience. J Med Virol 2020;92(7):814-818. DOI: 10.1002/ jmv.25801. 